\title{
Diagnosis and Treatment of Paroxysmal Sympathetic Hyperactivity in Medical ICU, University of Gondar Hospital, Northwest Ethiopia: A Case Report
}

This article was published in the following Dove Press journal: International Medical Case Reports Journal

\author{
Nebiyu Bekele (D) \\ Nebiyu Mesfin' \\ Tigest Hailu (D) ${ }^{2}$ \\ Abilo Tadesse ${ }^{1}$
}

'Department of Internal Medicine, College of Medicine and Health Sciences, University of Gondar, Gondar, Ethiopia;

${ }^{2}$ Department of Radiology, College of Medicine and Health Sciences, University of Gondar, Gondar, Ethiopia
Correspondence: Abilo Tadesse Department of Internal Medicine, College of Medicine and Health Sciences, University of Gondar, Gondar, Ethiopia Email abilo.tadesse@yahoo.com
Background: Paroxysmal sympathetic hyperactivity (PSH) is a neurologic syndrome characterized by paroxysmal and simultaneous occurrence of hypertension, hyperpyrexia, tachycardia, tachypnea, diaphoresis and dystonic posturing due to surge in sympathetic outflow after acquired brain injuries. Diagnosis of PSH is made using the paroxysmal sympathetic hyperactivity-assessment measure (PSH-AM) score, which comprises "clinical features severity" (CFS) score and "diagnosis likelihood tool" (DLT) score.

Case presentation: A 35-year-old woman diagnosed to have echo-proven chronic rheumatic heart disease for 25 years. Percutaneous balloon mitral valvotomy was done 6 weeks previously for severe mitral stenosis. Left atrial thrombus was detected after the procedure and anticoagulant (warfarin) was initiated. She presented with severe headache and repeated vomiting of 1 day duration on arrival to the hospital. She had frequent seizure attacks with subsequent loss of consciousness on third day of admission. Diagnosis of status epilepticus secondary to intracranial hemorrhage due to warfarin toxicity was made after CT-scan revealed acute subdural hematoma and ventricular bleeding. Then she was transferred to medical intensive care unit (ICU), intubated and put on mechanical ventilator. Anti-epileptic drugs, antibiotics, vitamin K and fresh frozen plasma were given. She developed paroxysms of hypertension, tachycardia, tachypnea, hyperpyrexia, diaphoresis and decerebrate posturing after 7 days of neurological insult. She had normal inter-ictal EEG tracing during cyclic autonomic surge. CFS score was 11 and DLT score was 10. In sum, PSH-AM score was 21, suggested "probable" diagnosis of PSH. Morphine, diazepam, propranolol and gabapentin were given in combination to treat PSH. Severity of autonomic storm started to improve on second week of ICU admission. On the third week of admission, her clinical condition deteriorated suddenly, she developed asystole and died of cardiac arrest despite cardiopulmonary resuscitation (CPR).

Conclusion: 'Clinical scoring' was used used to diagnose PSH, since there was no any confirmatory test. Cocktail of drugs were required to treat catecholamine surge in PSH.

Keywords: paroxysmal sympathetic hyperactivity; PSH, intracranial hemorrhage, warfarin toxicity

\section{Background}

Paroxysmal sympathetic hyperactivity (PSH) was first described as 'diencephalic autonomic epilepsy' by Wilder Penfield in 1929. ${ }^{1}$ The term "paroxysmal sympathetic hyperactivity" was first coined by Alejandro Rabinstein in 2007. ${ }^{2}$ It is a neurologic syndrome characterized by paroxysmal and simultaneous occurrence of 
hypertension, tachycardia, tachypnea, hyperpyrexia, diaphoresis and abnormal motor posturing due to surge in sympathetic outflow after acquired brain injuries. Almost all $(95 \%)$ cases of PSH are caused by traumatic head injury, anoxia and stroke. ${ }^{2-7,11,12}$ Since there is no confirmatory test, diagnosis of PSH is made using combination of "clinical features severity" (CFS) score and "diagnosis likelihood tool" (DLT) score, named as PSH-AM (assessment measure) score. The CFS score assesses presence and severity of clinical features, while the DLT measures the presence of compatible clinical parameters (frequency, duration, paroxysms and simultaneity of hyper-sympathetic episodes, antecedent cause, triggering factors, allodynia, excluding alternative causes, and response to treatment). PSH contributes to troublesome clinical effects including worst clinical outcome, physical disability, prolonged hospital stay and high cost for health care. ${ }^{6-10}$ Here, we discuss a case of PSH secondary to intracranial hemorrhage after warfarin toxicity.

\section{Case Presentation}

A 35-year-old woman diagnosed to have echocardiographic-proven chronic rheumatic heart disease (severe mitral stenosis with pulmonary hypertension) for the past 25 years. She had undergone percutaneous balloon mitral valvotomy (PBMV) 6 weeks previously.The left atrial thrombus was detected after the procedure and she started warfarin $5 \mathrm{mg}$, po, daily for 4 weeks and escalated to $7.5 \mathrm{mg}$ po daily to meet desired INR.

On her day of presentation to Emergency Medical OPD, she had a severe headache and repeated episodes of vomiting of ingested matter of 1 day duration. She gave recent history of nasal bleeding, and stopped warfarin by herself 3 days ago. She gave history of fever, malaise and generalized weakness of 1 week duration.

On initial clinical evaluation, vital signs were within normal limits and stable. She had no pallor of conjunctivae and had wet buccal mucosa. On cardiovascular examination, she had accentuated P2, mid-diastolic and pan-systolic murmur at mitral area, but no summation gallop. No cranial nerve palsy, no motor or sensory deficit, and meningeal irritation signs were negative. Findings in other systems were unremarkable. Diagnoses of chronic rheumatic heart disease (mitral valve disease with pulmonary hypertension), PBMV, left atrial thrombus, pyogenic meningitis R/o subarachnoid hemorrhage were made.

Laboratory values revealed white blood cell count (wbc) $=25,000 / \mu \mathrm{L}$ (neutrophil count $=85 \%$, lymphocyte count $=10 \%$ ), hemoglobin $(\mathrm{hgb})=12 \mathrm{gm} / \mathrm{dl}$, Platelets $=350,000 / \mu \mathrm{L}$. Liver function tests (LFTs) and renal function tests (RFTs) were within normal values. Blood culture was taken, but no growth at first, second or seventh day of incubation. Coagulation profiles showed prothrombin time $(\mathrm{PT})=35 \mathrm{sec}$ (normal value $=11-13 \mathrm{sec})$, partial thromboplastin time $($ PTT $)=53 \mathrm{sec}$ (normal value $=22-35 \mathrm{sec}$ ) and international normalized ratio $(I N R)=2.5 \quad$ (normal value $=0.8-1.4) \quad$ (Table-1). A lumbar puncture was deferred due to higher INR value. Electrocardiography (ECG) tracing showed P-mitrale, right bundle branch block (RBBB), and ST depression and inverted $\mathrm{T}$ wave at V1-V3, suggestive of left atrial enlargement and right ventricular strain. Trans-thoracic echocardiography revealed moderate mitral stenosis, mild mitral regurgitation, and severe pulmonary hypertension. No vegetation or

Table I Laboratory Results in Emergency Medical OPD (EMOPD) and Medical Intensive Care Unit (M-ICU)

\begin{tabular}{|c|c|c|c|}
\hline Variables & EMOPD & $\begin{array}{l}\text { M- } \\
\text { ICU }\end{array}$ & $\begin{array}{l}\text { Ref. } \\
\text { Value }\end{array}$ \\
\hline \multicolumn{4}{|l|}{ Complete blood count } \\
\hline Hemoglobin (hgb) & 12 & 9 & $12-18$ \\
\hline WBC $\left(\times 10^{3} / \mu \mathrm{L}\right)$ & 25 & 34 & $4-11$ \\
\hline Platelets $\left(\times 10^{3} / \mu \mathrm{L}\right)$ & 350 & 550 & $150-450$ \\
\hline ESR $(\mathrm{mm} / \mathrm{hr})$ & 75 & - & $\mathrm{I}-25$ \\
\hline \multicolumn{4}{|l|}{ Renal function tests } \\
\hline BUN (mg/dl) & 15 & 19 & $7-20$ \\
\hline $\mathrm{Cr}(\mathrm{mg} / \mathrm{dl})$ & 0.9 & I.I & $0.6-1.2$ \\
\hline \multicolumn{4}{|l|}{ Liver biochemical tests } \\
\hline ALT (U/L) & 30 & 33 & $0-35$ \\
\hline AST (U/L) & 43 & 50 & $0-35$ \\
\hline Alkaline phosphatase (U/L) & 75 & 95 & $50-250$ \\
\hline Bilirubin (mg/dl) & 0.3 & 0.7 & $0.3-1.0$ \\
\hline Total protein (gm/dl) & 6.5 & 6.0 & $6.0-8.0$ \\
\hline Albumin (gm/dl) & 4.8 & 3.2 & $3.5-5.5$ \\
\hline \multicolumn{4}{|l|}{ Coagulation profiles } \\
\hline Prothrombin time $(\mathrm{Sec})$ & 35 & 49.8 & $11-13$ \\
\hline Partial thromboplastin time $(\mathrm{Sec})$ & 53 & 63.2 & $22-35$ \\
\hline INR & 2.5 & 4.38 & $0.8-1.4$ \\
\hline \multicolumn{4}{|l|}{ Electrolytes } \\
\hline Potassium (meq/L) & 3.6 & 4.0 & $3.5-5.0$ \\
\hline Sodium (meq/L) & 137 & 130 & $135-145$ \\
\hline Chloride (meq/L) & 110 & 115 & $102-109$ \\
\hline \multicolumn{4}{|l|}{ Thyroid function tests } \\
\hline Serum TSH (mu/L) & - & 3.1 & $0.5-4.7$ \\
\hline Serum $T_{4}(\mu g / d)$ & - & 9.7 & $4.5-10.9$ \\
\hline Serum $T_{3}(\mathrm{ng} / \mathrm{dl})$ & - & 105 & $60-181$ \\
\hline
\end{tabular}

Note: NB: EMOPD, admission at Emergency Medical OPD; MICU, admission at Medical ICU. 
thrombus was seen. She was initiated with Ceftriaxone 2gm, IV, twice daily and Vancomycin $1 \mathrm{gm}, \mathrm{IV}$, twice daily, and warfarin was already discontinued.

On the third day of admission, she developed sudden onset, frequent tonic-clonic convulsive episodes with subsequent loss of consciousness. On physical examination, blood pressure $(B P)=130 / 90 \mathrm{mmhg}$, pulse rate $(\mathrm{PR})=116$ beats per minute $(\mathrm{bpm})$, respiratory rate $(\mathrm{RR})=32$ breaths per minute $(\mathrm{bpm})$, temperature $\left(\mathrm{T}^{0}\right)=38.60 \mathrm{c}$. There was transmitted sound all over the chest. A neurological examination revealed Glasgow Coma Score (GCS) of 8/15 (E2, M4, V2). Both eyes deviated to the left side. Pupils were mid-sized, but poorly reactive to light. She withdrew to painful stimuli. Meningeal irritation signs were negative. Diagnosis of status epilepticus secondary to intracranial hemorrhage due to warfarin toxicity was made after noncontrast brain CT scan revealed left-sided acute subdural hematoma and ventricular bleeds (Figures 1 and 2). Then, patient was initiated on diazepam, $10 \mathrm{mg} I \mathrm{~V}$, and Phenytoin $1 \mathrm{gm}$ IV bolus followed by 100mg IV 3 times a day to arrest the seizure. She was given $\mathrm{O}_{2}$ via face mask, NGtube was inserted, and urinary catheter was put in situ.

She was transferred to ICU after a diagnosis of status epilepticus, was intubated and put on a mechanical ventilator.

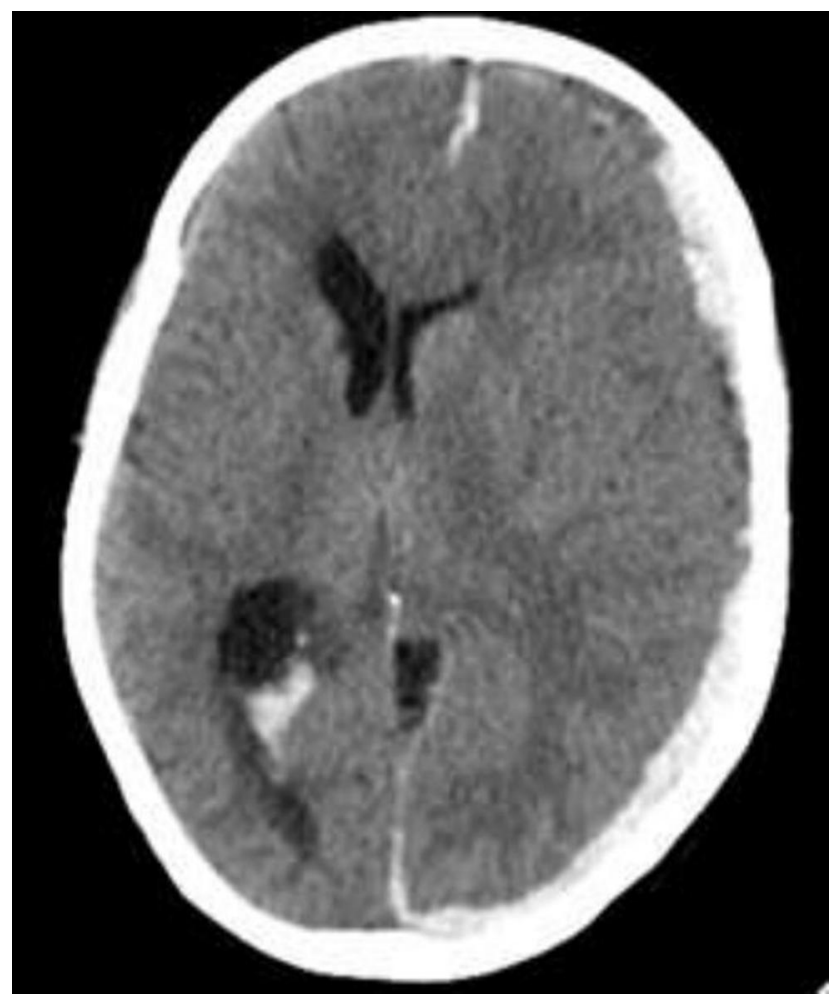

Figure I Left subdural hematoma with mass effect on underlying brain parenchyma and midline shift, and ventricular hemorrhage.

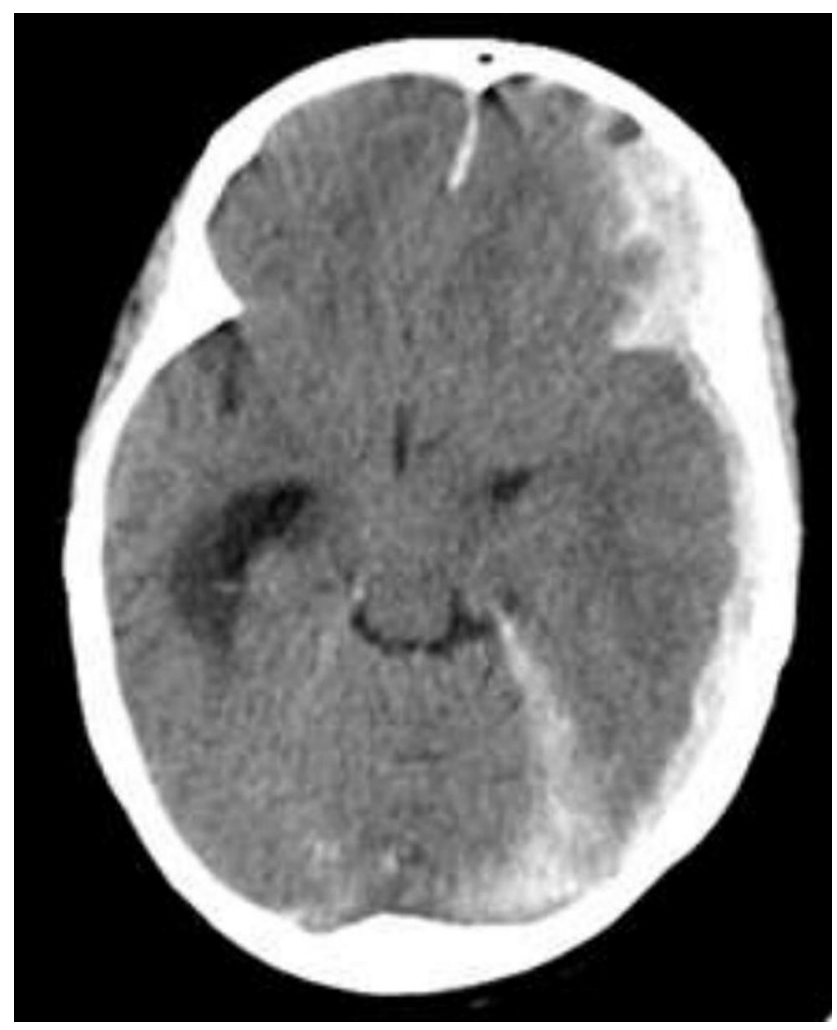

Figure 2 Left subdural hematoma with extension of the collection to lateral ventricles and dural reflections.

Vital signs and $\mathrm{Spo}_{2}$ were monitored by cardiac telemetry. Fresh frozen plasma 4 units, and vitamin k, $10 \mathrm{mg}$ IV once daily for 3 days were given. IV ceftriaxone and vancomycin were continued. Mannitol (20\%), 50gm IV bolus and 25gm IV 3 times daily was provided to decrease increased intracranial pressure (ICP). Valproic acid, 400mg po 3 times daily was added as the seizure was poorly controlled. A craniotomy was done by neurosurgeon on the same day of neuroimaging and evacuated clots of blood.

Repeat laboratory values revealed $\mathrm{WBC}=34,000 / \mu \mathrm{L}$ (neutrophil count $=85 \%$, lymphocyte count $=10 \%$ ), $\mathrm{hgb}=9 \mathrm{gm} / \mathrm{dl}$, platelets $=550,000 / \mu \mathrm{L}$. Coagulation profiles showed PT=49.8 $\sec (>3.5 \mathrm{x}$ ULN), PTT=63.2 $\mathrm{sec}(>1.5 \mathrm{x}$ ULN) and INR=4.38 ( $>3 x$ ULN). Serum LFTs, RFTs and electrolytes were with in normal limits (Table 1).

Paroxysms of increase in pulse rate, blood pressure, respiratory rate, temperature, sweating, and decerebrate posturing became typical after 7 days of neurological insult. It occurred while suctioning, body turning, and physical examination. It occurred at least 2-4 times a day, each persisting for between 5 and 40 minutes. PSH was considered by neurologist after repeated recordings of normal inter-ictal EEG tracing during episodes of autonomic surge. CSF score was 11 (heart rate $>140$ beats per minute, respiratory 
rate $=24-29$ breaths per minute, systolic blood pressure $=140$ 159 mmhg, temperature $=38-38.9$ 0c, mild sweating, and moderate posturing during episodes). DLT score was 11 (antecedent acquired brain injury, sympathetic surges were paroxysmal, simultaneous, episodic and episodes occurred $>2$ times daily and persisted for $>2$ weeks post-brain injury, provoked by non-painful stimuli (allodynia), and medications reduced sympathetic features). ${ }^{4,6-9}$ "Probable" diagnosis of PSH was made after patient scored 21 using PSH-AM score (sum of CFS score and DLT score). Then, she was adequately rehydrated with isotonic fluids; and started on morphine $2 \mathrm{mg}$, IV, as required (prn); paracetamol $1 \mathrm{gm}$, po as required (prn); propranolol, $40 \mathrm{mg}$ po 3 times a day; diazepam 5 mg, IV 3 times a day; and gabapentin 150-300 mg po 3 times a day. Her GCS started to improve on second week of ICU admission, and frequency and severity of sympathetic storm declined. IV antibiotics were discontinued after 2 weeks of therapy. On the third week of admission, her clinical condition deteriorated all of a sudden, developed asystole, and died of cardiac arrest despite cardiopulmonary resuscitation (CPR).

\section{Discussion}

PSH is a syndrome recognized in a subgroup of survivors of severe acquired brain injury, of simultaneous, paroxysmal transient increases in sympathetic (elevated heart rate, blood pressure, respiratory rate, temperature, sweating) and motor (posturing) activity. ${ }^{4}$

$\mathrm{PSH}$ is prevalent in young men, and incidence ranges from $8-33 \%$. Higher incidence was reported in those with diffuse traumatic brain injury. Traumatic brain injury $(80 \%)$, anoxic brain injury (10\%), and cerebrovascular accidents (5\%) took the majority of the share as etiology of PSH. The remaining $5 \%$ is caused by brain tumor, encephalitis, hydrocephalus and unspecified causes. ${ }^{2-7,11,12}$

Although pathophysiologic basis for PSH is incompletely understood, speculative explanations rest on "disconnection theory" and "excitation-inhibition ratio (EIR) model." Disconnection theory suggested a loss of inhibitory cortical centers control over caudal excitatory autonomic centers, while the EIR model proposed failure to modulate spinal cord excitatory sensory circuits by inhibitory centers within brainstem (the periaqueductal grey matter). ${ }^{5-8}$

PSH manifests abruptly in cyclic episodes, either spontaneously or in response to unavoidable non-noxious stimuli, such as tracheal suctioning, urinary catheterization, and body positioning. It usually occurs 1 week after acquired brain injury. Paroxysms appear 3 to 5 times a day and each episode on average lasts for 30 minutes to 1 hour. Diagnosis of PSH is made by combination of "CFS" score and "DLT" score, named as PSH-AM (assessment measure) score. ${ }^{6-10}$

Our patient presented with cyclic sympathetic storm with PSH-AM score of 21 after acquired brain injury, suggested "probable" diagnosis of PSH. Differential diagnosis for a patient presenting with altered mental status, muscle rigidity, hyperpyrexia and autonomic instability includes malignant hyperthermia, neuroleptic malignant syndrome, narcotic withdrawal syndrome, thyroid storm and encephalitis. Delayed onset of symptoms ( $\sim 1$ week) after exposure to anesthetic drugs; lack of exposure to neuroleptic drugs and narcotics; absence of preceding thyrotoxicosis features and normal thyroid function tests (TFTs); and CT-evidenced intracranial hemorrhage exclude likely differential diagnoses.

Uncontrolled PSH can lead to secondary brain injury with a worse GCS score, cardiac damage including arrhythmia and cardiomyopathy, and immunosuppression. It has been associated with increased infectious episodes, prolong hospital stay, worse functional independence, and higher health care costs. Although PSH was not a life threatening problem, it contributed to the death of this patient possibly due to arrhythmia with underlying cardiac disease. ${ }^{6-8}$

Medical treatments for PSH include combinations of $\mu$-opioid receptor agonist (morphine), non-selective ß-receptor blocker (propranolol), $\alpha_{2}$-receptor agonist (clonidine), GABA-receptor agonist (gabapentin), dopaminereceptor antagonist (bromocriptine) and benzodiazepines (diazepam) to abort or minimize PSH episodes via inhibition of sympathetic flow, afferent sensory process and effector end organ response. ${ }^{6-8,11-14}$ Early diagnosis and optimized treatment is believed to shorten ICU stay, facilitate patient recovery and minimize physical disability. In conclusion, 'clinical scoring' was used to dignose PSH, since there was no any confirmatory test. Cocktail of drugs were required to treat catecholamine surge in PSH.

\section{Abbreviations}

BP, blood pressure; CT scan, computerized tomogram; CFS, clinical features severity; CPR, cardiopulmonary resuscitation; DLT, diagnosis likelihood tool; ECG, electrocardiogram; EEG, electroencephalogram; EMV, eye opening, motor response, verbal response; ESR, erythrocyte sedimentation rate; Hgb, hemoglobin; MICU, medical intensive care unit; ICP, intracranial pressure; INR, international normalized ratio; IV, intravenous; LFT, liver function test; MU, 
million units; $\mathrm{O}_{2}$, oxygen; OPD, outpatient department; PR, pulse rate; $\mathrm{PO}$, per os; $\mathrm{PSH}$, paroxysmal sympathetic hyperactivity; PBMV, percutaneous balloon mitral valvotomy; PSH-AM, PSH-assessment measure; PT, prothrombin time, PTT, partial thromboplastin time; RR, respiratory rate, RBBB, right bundle branch block; RFT, renal function test; $\mathrm{Spo}_{2}$, arterial $\mathrm{O}_{2}$ saturation; $\mathrm{T}^{0}$, temperature; $\mathrm{WBC}$, white blood cell.

\section{Ethics Approval and Consent to Participate}

The authors declare that ethics approval was not required for this case report as we did not use any new procedures or any treatment, which was not approved for clinical use in our institution.

\section{Consent for Publication}

Written informed consent was obtained from the next-ofkin for publication of the case report and any accompanying images, since the patient died on the third week of admission in the hospital.

\section{Acknowledgment}

We are grateful to thank health personnel, who were taking care of the patient.

\section{Author Contributions}

All authors made substantial contributions to conception and design, acquisition of data, or analysis and interpretation of data; took part in drafting the article or revising it critically for important intellectual content; agreed to submit to the current journal; gave final approval of the version to be published; and agree to be accountable for all aspects of the work.

\section{Disclosure}

The authors declare that they have no competing interests.

\section{References}

1. Penfield W. Diencephalic autonomic epilepsy. Arch Neurol Psychiatry. 1929;22:358-374. doi:10.1001/archneurpsyc.1929.02220 020174010

2. Rabinstein AA. Paroxysmal sympathetic hyperactivity in the neurological intensive care unit. Neurol Res. 2007;29:680-682. doi:10.1179/016164107X240071

3. Perkes I, Baguley IJ, Nott MT, Menon DK. A review of paroxysmal sympathetic hyperactivity after acquired brain injury. Ann Neurol. 2010;68:126-135. doi:10.1002/ana.22066

4. Baguley IJ, Perkes IE, Fernandez-Ortega J-F, Rabinstein AA, Dolce G, Hendricks HT; Consensus Working Group. Paroxysmal sympathetic hyperactivity after acquired brain injury: consensus on conceptual definition, nomenclature, and diagnostic criteria. $J$ Neurotrauma. 2014;31:1515-1520. doi:10.1089/neu.2013.3301

5. Hinson HE, Puybasset L, Weiss N, et al. Neuroanatomical basis of paroxysmal sympathetic hyperactivity: a diffusion tensor imaging analysis. Brain Inj. 2015;29:455-461. doi:10.3109/02699052.2014. 995229

6. Choi HA, Jeon SB, Samuel S, Allison T, Lee K. Paroxysmal sympathetic hyperactivity after acute brain injury. Curr Neurol Neurosci Rep. 2013;13(8):370-379. doi:10.1007/s11910-013-0370-3

7. Meyfroidt G, Baguley IJ, Menon DK. Paroxysmal sympathetic hyperactivity: the storm after acute brain injury. Lancet Neurol. 2017;16:721-729. doi:10.1016/S1474-4422(17)30259-4

8. Godoy DA, Panhke P, Suarez PDG, Murillo-Cabezas F. Paroxysmal sympathetic hyperactivity: an entity to keep in mind. Med Intensiva. 2019;43(1):35-43. doi:10.1016/j.medin.2017.10.012

9. Hughes JD, Rabinstein AA. Early diagnosis of paroxysmal sympathetic hyperactivity in the ICU. Neuro Crit Care. 2014;20(3):454459.

10. Godo S, Irino S, Nakagawa A, et al. Diagnosis and management of paroxysmal sympathetic hyperactivity following acute brain injuries using a consensus-based diagnostic tool: a single institution case series. Tohoku J Exp Med. 2017;243(1):11-18. doi:10.1620/tjem. 243.11

11. Liu Y, Jolly S, Pokala K. Prolonged paroxysmal sympathetic storming associated with spontaneous subarachnoid hemorrhage. Case Rep Med. 2013;ID 358182. doi:10.1155/2013/358182

12. Di Luca DG, Mohney N, Kottapally M. Paroxysmal sympathetic hyperactivity with dystonia following bilateral thalamic and cerebellar hemorrhage: a case report. Neurology. 2018;90(Suppl):15.

13. Zheng R-Z, Lei Z-Q, Yang R-Z, Huang G-H, Zhang G-M. Identification and management of paroxysmal sympathetic hyperactivity after traumatic brain injury. Front Neurol. 2020;11(81):1-14. doi:10.3389/fneur.2020.00081

14. Samuel S, Allison TA, Lee K, Choi HA. Pharmacologic management of paroxysmal sympathetic hyperactivity after brain injury. $J$ Neurosci Nurs. 2016;48(2):82-89. doi:10.1097/JNN.000000000000 0207

International Medical Case Reports Journal

Dovepress

\section{Publish your work in this journal}

The International Medical Case Reports Journal is an international, peer-reviewed open-access journal publishing original case reports from all medical specialties. Previously unpublished medical posters are also accepted relating to any area of clinical or preclinical science. Submissions should not normally exceed 2,000 words or 4

published pages including figures, diagrams and references. The manuscript management system is completely online and includes a very quick and fair peer-review system, which is all easy to use. Visit http://www.dovepress.com/testimonials.php to read real quotes from published authors. 